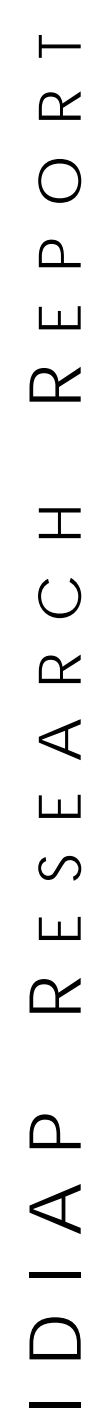

ш

ง

ш

ฯ

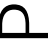

Dalle Molle Institute for Perceptual Artificial Intelligence • P.O.Box 592 • Martigny $\bullet$ Valais $\bullet$ Switzerland

phone $+41-27-721 \quad 77 \quad 11$

fax $\quad+41-27-721 \quad 77 \quad 12$

e-mail secretariat@idiap.ch internet http://www.idiap.ch

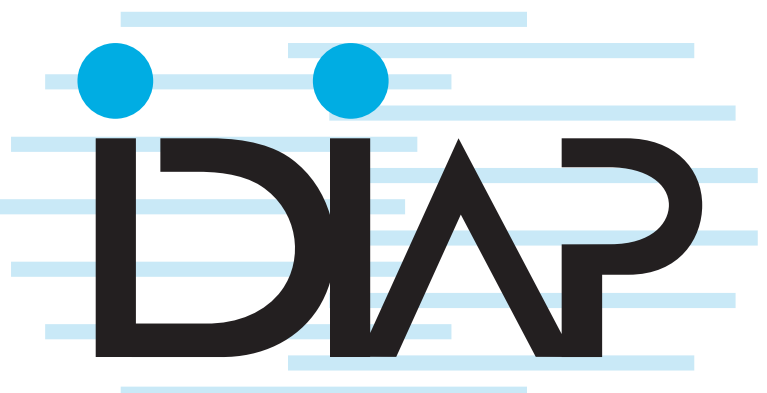

Combining Neural Gas and

LEARNING VECTOR

QuANTIZATION FOR CURSive

Character Recognition

Francesco Camastra ${ }^{a}$

Alessandro Vinciarelli ${ }^{b}$

IDIAP-RR 01-18

JUNE 2001

TO APPEAR IN

Neurocomputing a INFM - Computer Science Department (DISI), University of Genova - Via Dodecaneso 35 - 16146 Genova (Italy) - e-mail: camastra@disi.unige.it

b IDIAP - Institut Dalle Molle d'Intelligence Artificielle Perceptive - Rue du Simplon 4, CP592 - 1920 Martigny, Switzerland - e-mail: alessandro.vinciarelli@idiap.ch 



\title{
Combining Neural Gas and Learning Vector Quantization for Cursive Character RECOGNITION
}

\author{
Francesco Camastra Alessandro Vinciarelli
}

JunE 2001

TO APPEAR IN

Neurocomputing

\begin{abstract}
This paper presents a cursive character recognizer, a crucial module in any Cursive Script Recognition system based on a segmentation and recognition approach.

The character classification is achieved by combining the use of Neural Gas (NG) and Learning Vector Quantization (LVQ). NG is used to verify whether lower and upper case version of a certain letter can be joined in a single class or not. Once this is done for every letter, it is possible to find an optimal number of classes maximizing the accuracy of the LVQ classifier.

A database of 58000 characters was used to train and test the models. The performance obtained is among the highest presented in the literature for the recognition of cursive characters.
\end{abstract}




\section{Introduction}

Off-line Cursive Script Recognition (CSR) has several industrial applications such as the reading of postal addresses and the automatic processing of forms, checks and faxes (Steinherz et al., 1999; Plamondon and Srihari, 2000). Among other CSR approaches (Senior and Robinson, 1998; Kim and Govindaraju, 1997) one attempts to segment words into letters (Bozinovic and Srihari, 1989; Edelman et al., 1990). Since no method is available to achieve a perfect segmentation, a word is first oversegmented, i.e. fragmented into primitives that are characters or parts of them (a perfect segmentation into letters is extremely difficult), then neighboring primitives are joined together in all possible combinations (a limit on the number of consecutive fragments that can form a character is usually experimentally determined).

Given a combination where $n$ aggregations of primitives appear, a matching score with all the $n$ letter long words in the lexicon is calculated. A common way to calculate it is to average over the scores of classifying each aggregation of primitives as the corresponding letter of the lexicon entry under examination (see fig. 1). The word with the optimal score is found by applying Dynamic Programming techniques (Bellman and Dreyfus, 1962).

The role of the cursive character recognizer in the above described architecture is crucial. It has to cope with the high variability of the cursive letters and their intrinsic ambiguity (letters like $e$ and $l$ or $u$ and $n$ can have the same shape). In this paper we present a cursive character recognizer combining the use of Neural Gas (NG) and Learning Vector Quantization (LVQ). The NG is used to verify when the upper and lower case versions of a letter can form a common class. This happens when the two characters (e.g. $o$ and $O$ ) are similar in shape and their vectors in the feature space occupy neighboring or even overlapping regions. By grouping the characters in this way, the number of classes is reduced and a more suitable representation of the data is obtained. The classifier, based on LVQ, provides for the aggregation of primitives not only a simple attribution to a given class $C$ but also a score that is the euclidean distance between the feature vector extracted from the aggregation and the closest $C$ codevector.

This paper is organized as follows: in Section 2 the method for extracting features for character representation is presented; a review of LVQ and NG is provided in Section 3 and 4 respectively; in Section 5 reports some experimental results; in Section 6 some conclusions are drawn.

\section{Feature Extraction}

Most character recognizers do not work on the raw image, but on a suitable compact representation of the image by means of a vector of features. Since cursive characters present high variability in shapes, a feature extractor should have negligible sensitivity to local shifts and distortions. Therefore feature extractors that perform local averaging are more appropriate than others that yield an exact reconstruction of the pattern (e.g. Zernike polynomials, moments) as shown in (Camastra and Vinciarelli, 2001). The feature extractor, fed with the binary image of an isolated cursive character, generates local and global features. The local features are extracted from subimages (cells) arranged in a regular grid ${ }^{1}$ covering the whole image. A fixed set of operators is applied to each cell. The first operator is a counter that computes the percentage of foreground pixels in the cell (gray feature) with respect to the total number of foreground pixels in the character image. If $n_{i}$ is the number of foreground pixels in cell $i$ and $M$ is the total number of foreground pixels in the pattern, then the gray feature related to cell $i$ is $\frac{n_{i}}{M}$.

The other operators try to estimate to which extent the black pixels in the cell are aligned along some directions. For each direction of interest, a set of $N$, equally spaced, straight lines are defined, that span the whole cell and that are parallel to the chosen direction. Along each line $j \in[1, N]$ the number $n_{j}$ of black pixels is computed and the sum $\sum_{i}^{N} n_{j}^{2}$ is then obtained for each direction. The difference

\footnotetext{
${ }^{1}$ Small translations of the input patterns can significantly change the distribution of the pixels across the cells. In order to smooth this effect, the cells are partially overlapped.
} 
between the sums related to orthogonal directions is used as feature. In our case the directions of interest were $0^{\circ}$ and $90^{\circ}$.

We enriched the local feature set with two global features giving information about the overall shape of the cursive character and about its position with respect to the baseline of the cursive word. As shown in figure 2, the baseline is the line on which a writer implicitly aligns the word in the absence of rulers. The first global feature measures the fraction of the character below the baseline and detects eventual descenders. The second feature is the width/height ratio.

The number of local features can be arbitrarily determined by changing the number of cells or directions examined in each cell. Since classifier reliability can be hard when the number of features is high (curse of dimensionality, (Bellman, 1961)), we use simple techniques for feature selection in order to keep the feature number as low as possible. Directional features corresponding to different directions were applied and the one having the maximal variance was retained. Therefore the feature set was tested changing the number of cells and the grid giving the best results $(4 \times 4)$ was selected.

In the reported experiments we used a feature vector of 34 elements. Two features are global (baseline and width/height ratio) while the remaining 32 are generated from 16 cells, placed on a regular $4 \times 4$ grid; from each cell, the gray feature and one directional feature are extracted.

\section{Learning Vector Quantization}

Learning Vector Quantization $(L V Q)$ is a supervised version of vector quantization and generates codevectors to produce "near-optimal decision boundaries" (Kohonen, 1997).

LVQ consists of the application of three consecutive different learning techniques, i.e. LVQ1, LVQ2, LVQ3 ${ }^{2}$. LVQ1 uses for classification the nearest-neighbour decision rule; it chooses the class of the nearest codebook vector.

LVQ1 learning is performed in the following way: if $\bar{m}_{t}^{c}{ }^{3}$ is the nearest codevector to the input vector $\bar{x}$, then

$$
\begin{array}{ll}
\bar{m}_{t+1}^{c}=\bar{m}_{t}^{c}+\alpha_{t}\left[\bar{x}-\bar{m}_{t}^{c}\right] & \text { if } \bar{x} \text { is classified correctly } \\
\bar{m}_{t+1}^{c}=\bar{m}_{t}^{c}-\alpha_{t}\left[\bar{x}-\bar{m}_{t}^{c}\right] & \text { if } \bar{x} \text { is classified incorrectly } \\
\bar{m}_{t+1}^{i}=\bar{m}_{t}^{i} & i \neq c
\end{array}
$$

where $\alpha_{t}$ is the learning rate at time $t$.

Since LVQ1 tends to push codevectors away from the decision surfaces of the Bayes rule, it is necessary to apply to the codebook generated a successive learning technique called LVQ2.

LVQ2 tries harder to approximate the Bayes rule by pairwise adjustments of codevectors belonging to adjacent classes. If $\bar{m}^{s}$ and $\bar{m}^{p}$ are nearest neighbours of different classes and the input vector $\bar{x}$, belonging to the $\bar{m}^{s}$ class, is closer to $\bar{m}^{p}$ and falls into a zone of values called window ${ }^{4}$, the following rule is applied:

$$
\begin{aligned}
& \bar{m}_{t+1}^{s}=\bar{m}_{t}^{s}+\alpha_{t}\left[\bar{x}-\bar{m}_{t}^{s}\right] \\
& \bar{m}_{t+1}^{p}=\bar{m}_{t}^{p}-\alpha_{t}\left[\bar{x}-\bar{m}_{t}^{p}\right]
\end{aligned}
$$

Since the application of LVQ2 tends to overcorrect the class boundaries, it is necessary to include additional corrections that ensure that the codebook continues approximating the class distributions. In order to assure that, it is necessary to apply a further algorithm (LVQ3).

If $\bar{m}^{i}$ and $\bar{m}^{j}$ are the two closest codevectors to input $\bar{x}$ and $\bar{x}$ falls in the window, the following rule

\footnotetext{
${ }^{2}$ LVQ2 and LVQ3 were proposed, on empirical basis, in order to improve LVQ1 algorithm

${ }^{3} \bar{m}_{t}^{c}$ stands for the value of $\bar{m}^{c}$ at time $t$.

${ }^{4}$ The window is defined around the midplane of $\bar{m}^{s}$ and $\bar{m}^{p}$.
} 
is $\operatorname{applied}^{5}$ :

$$
\begin{array}{ll}
\bar{m}_{t+1}^{i}=\bar{m}_{t}^{i} & \text { if } C\left(\bar{m}^{i}\right) \neq C(\bar{x}) \wedge C\left(\bar{m}^{j}\right) \neq C(\bar{x}) \\
\bar{m}_{t+1}^{j}=\bar{m}_{t}^{j} & \text { if } C\left(\bar{m}^{i}\right) \neq C(\bar{x}) \wedge C\left(\bar{m}^{j}\right) \neq C(\bar{x}) \\
\bar{m}_{t+1}^{i}=\bar{m}_{t}^{i}-\alpha_{t}\left[\bar{x}_{t}-\bar{m}_{t}^{i}\right] & \text { if } C\left(\bar{m}^{i}\right) \neq C(\bar{x}) \wedge C\left(\bar{m}^{j}\right)=C(\bar{x}) \\
\bar{m}_{t+1}^{j}=\bar{m}_{t}^{j}+\alpha_{t}\left[\bar{x}_{t}-\bar{m}_{t}^{j}\right] & \text { if } C\left(\bar{m}^{i}\right) \neq C(\bar{x}) \wedge C\left(\bar{m}^{j}\right)=C(\bar{x}) \\
\bar{m}_{t+1}^{i}=\bar{m}_{t}^{i}+\alpha_{t}\left[\bar{x}_{t}-\bar{m}_{t}^{i}\right] & \text { if } C\left(\bar{m}^{i}\right)=C(\bar{x}) \wedge C\left(\bar{m}^{j}\right) \neq C(\bar{x}) \\
\bar{m}_{t+1}^{j}=\bar{m}_{t}^{j}-\alpha_{t}\left[\bar{x}_{t}-\bar{m}_{t}^{j}\right] & \text { if } C\left(\bar{m}^{i}\right)=C(\bar{x}) \wedge C\left(\bar{m}^{j}\right) \neq C(\bar{x}) \\
\bar{m}_{t+1}^{i}=\bar{m}_{t}^{i}+\epsilon \alpha_{t}\left[\bar{x}_{t}-\bar{m}_{t}^{i}\right] & \text { if } C\left(\bar{m}^{i}\right)=C\left(\bar{m}^{j}\right)=C(\bar{x}) \\
\bar{m}_{t+1}^{j}=\bar{m}_{t}^{j}+\epsilon \alpha_{t}\left[\bar{x}_{t}-\bar{m}_{t}^{j}\right] & \text { if } C\left(\bar{m}^{i}\right)=C\left(\bar{m}^{j}\right)=C(\bar{x})
\end{array}
$$

where $\epsilon \in[0,1]$ is a fixed parameter.

LVQ3 is self-stabilizing, i.e. the optimal placement of the codebook does not change while continuing learning.

\section{Neural Gas}

Neural Gas is an unsupervised version of vector quantization. In neural gas model, in contrast to SOM, no topology of a fixed dimensionality is imposed on the network . Neural Gas consists of a set of $M$ units : $A=\left(c_{1}, c_{2}, \ldots, c_{m}\right)$. Each unit $c_{i}$ has an associated reference vector $w_{c_{i}}\left(w_{c_{i}} \in R^{n}\right)$ indicating its position or receptive field center in input space. The learning algorithm of the neural gas is the following:

1. Initialize the set $A$ to contain units $c_{i}$, with $w_{c_{i}} \in R^{n}$, chosen randomly according to input distribution $p(\xi)$. Besides, initialize the time parameter $t$, to 0 .

2. Generate at random an input $\xi$ according to $p(\xi)$.

3. Order all elements of $A$ according to the distance of their reference vectors to $\xi$ e.g., find the sequence of indices $S=\left(i_{0}, i_{1}, \ldots, i_{M-1}\right)$ such that $w_{i_{0}}$ is the reference vector closest to $\xi, w_{i_{1}}$ is the second vector closest to $\xi$ etc. Let $k_{i}(\xi, A)$ the rank associated with $w_{i}{ }^{6}$.

4. Adapt the reference vectors according to :

$$
\Delta w_{i}=\epsilon(t) h_{\lambda}\left(k_{i}(\xi, A)\right)\left(\xi-w_{i}\right)
$$

where :

$$
\begin{gathered}
h_{\lambda}\left(k_{i}(\xi, A)\right)=\exp \left(-\frac{k_{i}}{\lambda(t)}\right) \\
\lambda(t)=\lambda_{i}\left(\frac{\lambda_{f}}{\lambda_{i}}\right)^{\frac{t}{t_{f}}} \\
\epsilon(t)=\epsilon_{i}\left(\frac{\epsilon_{f}}{\epsilon_{i}}\right)^{\frac{t}{t_{f}}}
\end{gathered}
$$

5. Increase the time parameter $t: t=t+1$

6. If $t<t_{f}$ continue with step 2 .

For the time dependent parameters suitable initial values $\lambda_{i}, \epsilon_{i}$ and final values $\lambda_{f}, \epsilon_{f}$ have to be chosen. For the above-mentioned parameter in our work, we adopted the values suggested in (Martinetz et al., 1993, 1994; Fritzke, 1997).

\footnotetext{
${ }^{5} C(\bar{q})$ stands for the class of $\bar{q}$.

${ }^{6} w_{i}$ stands for $w_{c_{i}}$. This convention is also adopted in the following formulae.
} 


\section{$5 \quad$ Experiments and Results}

The combined use of NG and LVQ is shown to improve the performance of a cursive character classifier. The letters are present in the database in both upper and lower case version. In some cases, the two versions are different and must be considered as separate classes. In some other cases, the two versions are similar and can be joined in a single class.

The NG is used to measure the overlapping in the feature space of the vectors corresponding to the two versions of each character. When the overlapping is high enough, upper and lower case versions of the letter are joined in a single class. This improves the performance of the LVQ over such classes and results in a better accuracy of the overall character classifier.

Subsection 5.1 describes the database used in the experiments, subsections 5.2 and 5.3 show how the optimal class representation was found and the recognition experiments respectively.

\subsection{The character database}

The cursive characters used to train and test the recognizer were extracted from the handwritten words belonging to two different data sets. The first one is the CEDAR ${ }^{7}$ database (Hull, 1994). The second one is a database of handwritten samples collected by the United States Postal Service. In both cases the data were collected in a postal plant by digitizing handwritten addresses.

The characters are extracted from the words through a segmentation process performed by the system in which the recognizer is embedded. Before being segmented, the words are desloped and deslanted following the scheme described in (Nicchiotti and Scagliola, 1999). The resulting character database contains 58000 elements. The letter distribution (shown in figure 3) reflects the prior distribution of the postal plants where the handwritten words were collected. For this reason, some letters are very frequent while others are almost absent.

The database is split with a random process into training, validation and test set containing respectively $\mathrm{x}, \mathrm{y}$ and $\mathrm{z}$ characters.

\subsection{Optimal number of classes finding}

Clustering allows to verify whether vectors corresponding to the upper and lower case versions of the same letter are distributed in neighboring regions of the feature space or not. The more the two versions of the letter are similar in shape the more their vectors are overlapping (e.g. like $o$ and $O$ ) and can be joined in a single class. On the other hand, when the two versions of a character are very different (e.g. $g$ and $G$ ), it is better to consider them as separate classes.

Clustering was performed by means of Neural Gas (NG) and Self Organizing Map (SOM). In Table 1 the performances of different SOM and NG maps, measured in terms of quantization error ${ }^{8}$ on the whole character database, are reported. Given a number of neurons, the NG performs always better than the SOM and is, for this reason, selected.

The quantization error can be reduced by increasing the number of nodes, but this leads to overfitting the map onto the training set, i.e. to decreasing its generalization properties. The map with 1300 neurons represents the best trade off between quantization and generalization error and is for this reason retained as optimal.

The neurons were labelled with a $\mathrm{kNN}$ technique ${ }^{9}$ and divided into 26 subsets collecting all the nodes showing at least one version of each letter $\alpha$ among the $k$ classes in the label. For each subset, the percentage $\eta_{\alpha}$ of nodes having upper and lower case versions of the letter $\alpha$ in the label was calculated. The results are reported (for every subset) in figure 4. The percentage is an index of the overlapping of the classes of the uppercase and lowercase versions of the letter. This information can be used to

\footnotetext{
${ }^{7}$ Center of Excellence in Document Analysis and Recognition, State University of New York at Buffalo (USA). All the words belonging to directories train/cities and train/states were used.

${ }^{8}$ Using the notation adopted in section 4 , the quantization error $Q_{E}$ is defined as follows: $Q_{E}=\sum_{j}^{N} \sum_{i}^{M}\left|\xi_{j}-w_{i}\right|^{2}$.

${ }^{9}$ Each node is labelled with the classes of the $k$ closest feature vectors.
} 
represent the data with a number of different classes ranging from 26 (uppercase and lowercase always joined in a single class) to 52 (uppercase and lowercase always in separate classes). For example a class number equal to 46 means that, for the six letters showing the highest values of $\eta$ (i.e. $c, x, o, w, y, z$ ) uppercase and lowercase versions are joined in a single class.

\subsection{Recognition experiments}

The percentage $\eta$ was used to look for the optimal number of classes. The letters showing the highest values of $\eta$ were represented by a single class containing both upper and lower case versions. We trained LVQ nets with different number of classes. In each trial, the number of codevectors and the learning rate were selected by means of cross-validation (Stone, 1974) and the learning sequence LVQ1+LVQ2+LVQ3 was adopted. The number of LVQ codevectors, assigned to each class, was proportional to the a-priori class probability.

In table 3 , for different class numbers, the performances on the test set, measured in terms of recognition rate in absence of rejection, are reported.

The performance is shown to be improved by decreasing the number of classes when this is higher than an optimal value (in this case 39). A further reduction of the number of classes results in a lower accuracy. The $\eta$ parameter is then reliable in estimating the optimal number of classes.

This is confirmed by looking at the performance of the recognizer over the single characters. Table 2 reports the change in recognition rate of each character when passing from 52 to 39 classes. The characters are ordered following the value of $\eta$. The first 13 classes show in most cases a significant accuracy improvement. The other classes show smaller changes in both positive and negative directions.

The effect on the overall performance of the recognizer is influenced by the letter distribution. In our case, the letters showing the highest improvements are not enough represented to significantly affect the overall accuracy, but in other cases, the distribution can be different and the improvement of the recognition rate much higher.

Our best result in terms of recognition rate is $84.52 \%$. The only result we know (Yamada and Nakano, 1996), obtained on a smaller test, is approximately $75 \%$. In fig. 5 the confusion matrix is shown. The cumulative probability function of the correct classification is reported in fig. 6 . The probabilities of classification of a character correctly top three and top twelve positions are respectively $95.76 \%$ and $99.50 \%$. In our opinion, the fundamental sources of misclassification for our classifier are two. The first one (for the most rare letters) is the low number of available samples. The second is the intrinsic ambiguity in cursive characters. In fact, some couples of letters (e.g. $e / l$ or $a / o$ ) are very difficult to be distinguished. This is confirmed by the confusion matrix and by the high recognition rate in the top three positions.

\section{Conclusion}

We have presented an isolated cursive character recognizer, a crucial module in Cursive Script Recognition systems based on a segmentation and recognition approach.

An improvement of the correct classification rate is obtained by combining the use of NG and LVQ. The NG allows to obtain a suitable representation of classes, LVQ performs the actual character recognition. The optimal representation of classes is obtained by evaluating the overlapping in the feature space of the vectors corresponding to upper and lower case versions of each letter. When the degree of overlapping is high enough, the two versions can be joined in a single class resulting in an improvement of the classifier performance.

The accuracy achieved is the highest presented, to our knowledge, in the literature. 


\section{References}

Bellman, R., 1961. Adaptive Control Processes: A Guided Tour. Princeton University Press.

Bellman, R., Dreyfus, S., 1962. Applied Dynamic Programming. Princeton University Press.

Bozinovic, R. M., Srihari, S. N., January 1989. Off-line cursive script word recognition. IEEE Transactions on Pattern Analysis and Machine Intelligence 11 (1), 69-83.

Camastra, F., Vinciarelli, A., May 2001. Cursive character recognition by Learning Vector Quantization. Pattern Recognition Letters 22 (6), 625-629.

Edelman, S., Flash, T., Ullman, S., March 1990. Reading cursive handwriting by alignment of letter prototypes. International Journal of Computer Vision 5 (3), 303-331.

Fritzke, B., April 1997. Some competitive learning methods. Tech. rep., Ruhr University Bochum.

Hull, J. J., May 1994. A database for handwritten text recognition research. IEEE Transactions on Pattern Analysis and Machine Intelligence 16 (5), 550-554.

Kim, G., Govindaraju, V., April 1997. A lexicon driven approach to handwritten word recognition for real time applications. IEEE Transactions on Pattern Analysis and Machine Intelligence 19 (4), $366-379$.

Kohonen, T., 1997. Self-Organizing Maps. Springer Verlag, Berlin.

Martinetz, T., , Schulten, K., 1994. Topology Representing Networks. Neural Networks 3, 507-522.

Martinetz, T., Berkovich, S., Schulten, K., 1993. "Neural Gas" network for vector quantization and its application to time-series prediction. IEEE Transactions on Neural Networks 4 (4), 558-569.

Nicchiotti, G., Scagliola, C., 1999. Generalised projections: a tool for cursive character normalization. In: Proceedings of $5^{t h}$ International Conference on Document Analysis and Recognition. IEEE Press.

Plamondon, R., Srihari, S., 2000. On-line and off-line handwriting recognition: A comprehensive survey. IEEE Transactions on Pattern Analysis and Machine Intelligence , 63-84.

Senior, A. W., Robinson, A. J., March 1998. An off-line cursive handwriting recognition system. IEEE Transactions on Pattern Analysis and Machine Intelligence 20 (3), 309-321.

Steinherz, T., Rivlin, E., Intrator, N., February 1999. Off-line cursive script word recognition - a survey. International Journal of Document Analysis and Recognition 2 (2), 1-33.

Stone, M., 1974. Cross-validatory choice and assessment of statistical prediction. Journal of the Royal Statistical Society 36 (1), 111-147.

Yamada, H., Nakano, Y., 1996. Cursive handwritten word recognition using multiple segmentation determined by contour analysis. IEICE Transactions on Informations and Systems 79 (5), 464-470. 

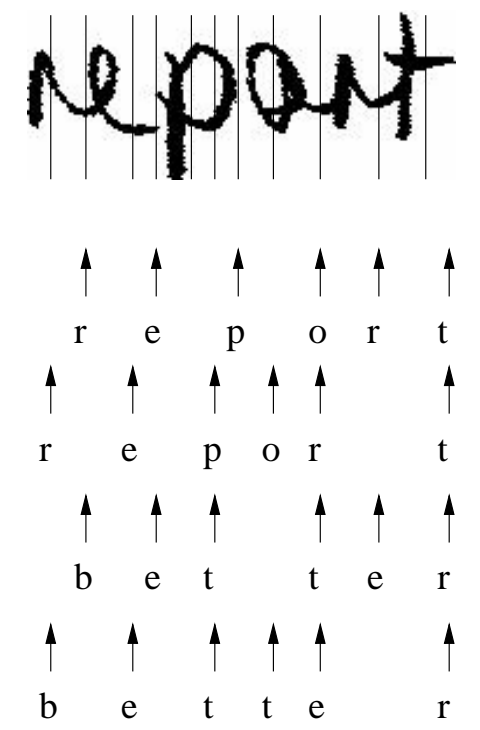

S1

S2

S3

S4

Figure 1: Score calculation. Different combinations of primitive aggregations. Each combination gives a different score. In our case, the score is the distance between the pattern enclosed by two arrows and the closest LVQ prototype labelled with the same characterer as the corresponding word letter. It is possible to have several combinations for the same word and several transcriptions of the same combination. 

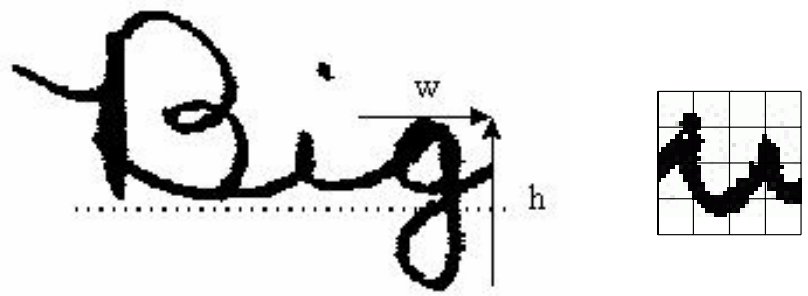

Figure 2: Global features. The dashed line is the baseline, the fraction of $h$ below is used as first global feature. The second global feature is the ratio $w / h$. 


\begin{tabular}{|c|c|c|}
\hline neurons & SOM(q.error) & NG (q.error) \\
\hline \hline 1300 & 0.197290 & 0.162117 \\
\hline 900 & 0.204126 & 0.167981 \\
\hline 600 & 0.209687 & 0.175800 \\
\hline 400 & 0.217515 & 0.182672 \\
\hline 200 & 0.227662 & 0.195116 \\
\hline 100 & 0.242464 & 0.208706 \\
\hline
\end{tabular}

Table 1: Quantization error of SOM and NG for different neuron numbers. 


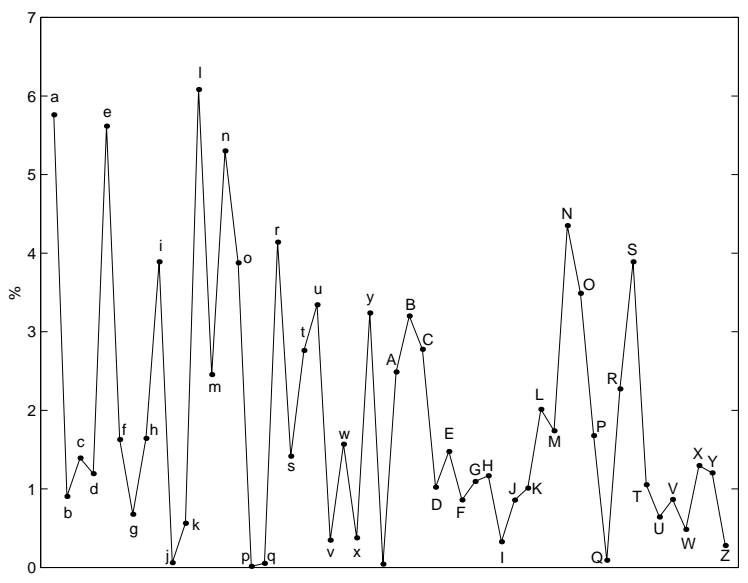

Figure 3: Letter distribution in the test set. 


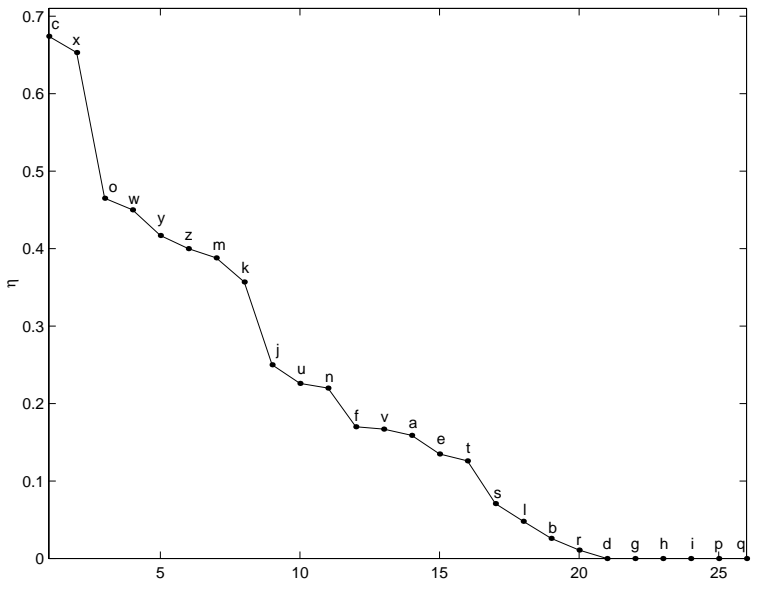

Figure 4: Value of $\eta$ for each letter. 


\begin{tabular}{|c|c|c|}
\hline class & 52 classes & 39 classes \\
\hline \hline $\mathrm{c}$ & 88.99 & 88.47 \\
\hline $\mathrm{x}$ & 84.40 & 88.23 \\
\hline $\mathrm{o}$ & 90.19 & 91.76 \\
\hline $\mathrm{w}$ & 71.39 & 77.60 \\
\hline $\mathrm{y}$ & 87.94 & 88.23 \\
\hline $\mathrm{z}$ & 65.90 & 85.71 \\
\hline $\mathrm{m}$ & 72.54 & 81.84 \\
\hline $\mathrm{k}$ & 59.86 & 62.25 \\
\hline $\mathrm{j}$ & 70.73 & 75.70 \\
\hline $\mathrm{u}$ & 90.55 & 90.55 \\
\hline $\mathrm{n}$ & 88.13 & 87.53 \\
\hline $\mathrm{f}$ & 80.50 & 80.71 \\
\hline $\mathrm{v}$ & 73.81 & 76.82 \\
\hline $\mathrm{a}$ & 83.62 & 84.01 \\
\hline $\mathrm{e}$ & 82.30 & 84.36 \\
\hline $\mathrm{t}$ & 87.94 & 90.55 \\
\hline $\mathrm{s}$ & 81.87 & 83.34 \\
\hline $\mathrm{l}$ & 83.25 & 82.22 \\
\hline $\mathrm{b}$ & 88.15 & 87.89 \\
\hline $\mathrm{r}$ & 85.80 & 83.34 \\
\hline $\mathrm{d}$ & 80.94 & 80.47 \\
\hline $\mathrm{g}$ & 80.58 & 80.00 \\
\hline $\mathrm{h}$ & 83.11 & 82.56 \\
\hline $\mathrm{i}$ & 75.98 & 75.46 \\
\hline $\mathrm{p}$ & 91.38 & 88.61 \\
\hline
\end{tabular}

Table 2: Change in the recognition rate when passing from 52 (lower and upper case versions of the letter never joined in a single class) to 39 classes (lower and upper case versions of the letter joined in a single class for the first 13 letters). The characters are ordered following the value of $\eta$.

\begin{tabular}{|c|c|}
\hline class number & performance \\
\hline \hline 52 & 83.74 \\
\hline 46 & 83.91 \\
\hline 42 & 84.25 \\
\hline 41 & 84.27 \\
\hline 39 & 84.52 \\
\hline 36 & 84.38 \\
\hline 26 & 84.27 \\
\hline
\end{tabular}

Table 3: Recognition rates on the Test Set, in absence of rejection, for several class numbers. 


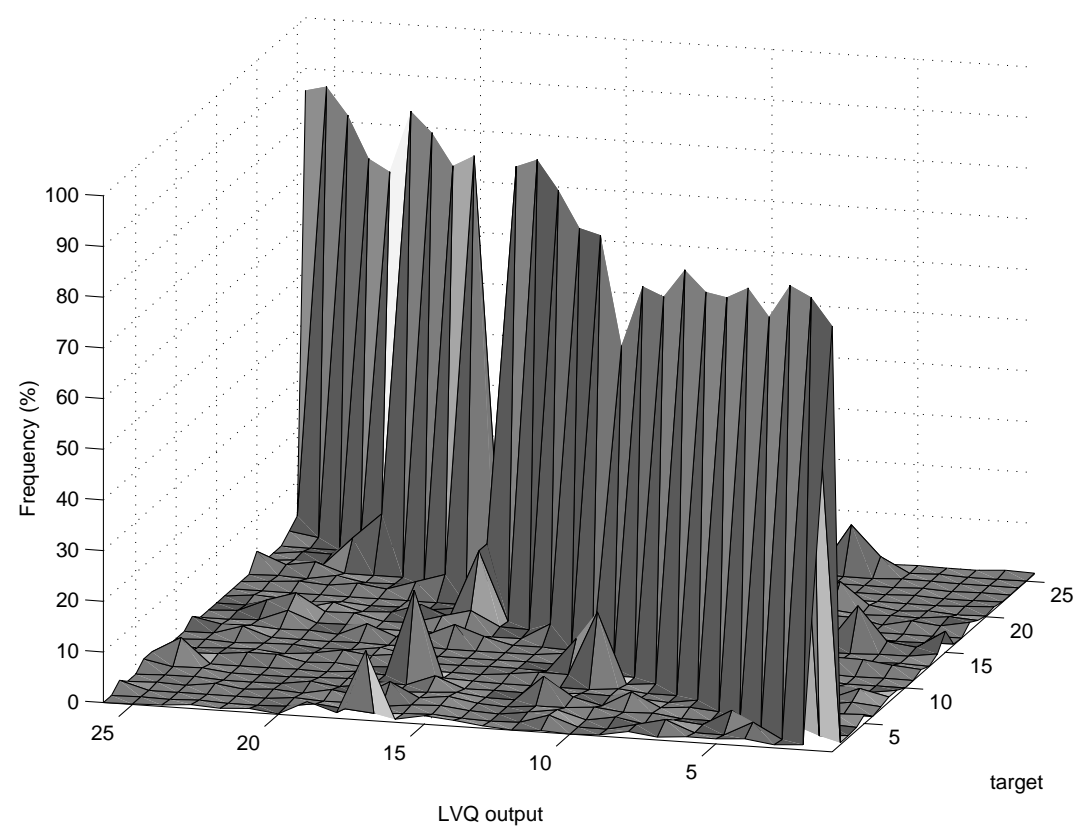

Figure 5: Confusion Matrix of the LVQ classifier. The letters are mapped to the numbers from 1 to 26. The letter $a$ is mapped to $1, b$ is mapped to $2, \ldots, z$ to 26 .

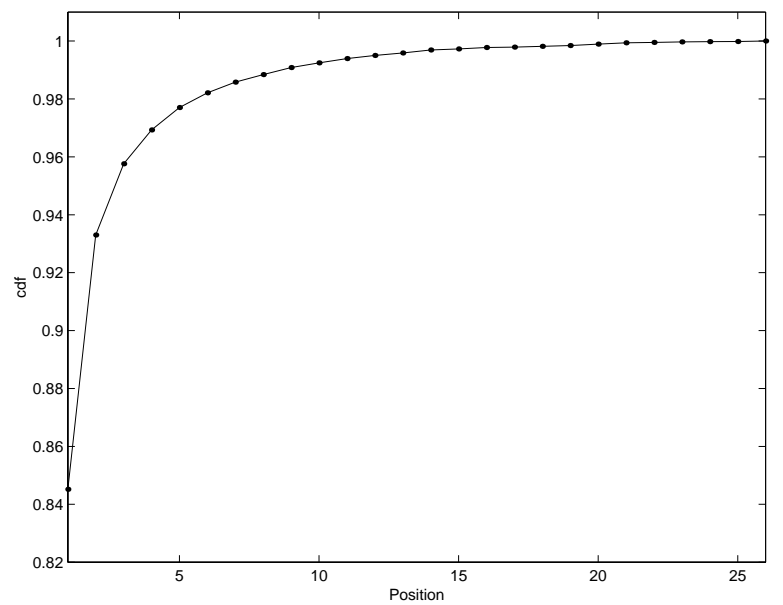

Figure 6: Cumulative probability function of the correct classification of LVQ classifier. 